\title{
Periocular and anterior orbital necrosis after upper eyelid gold weight loading: operation-related or self-inflicted?
}

This article was published in the following Dove Press journal:

Clinical Ophthalmology

30 April 2014

Number of times this article has been viewed

\author{
Roy Schwartz' \\ Ran Ben Cnaan' \\ Ophir Schein² \\ Michael Giladi ${ }^{3}$ \\ Michal $\mathrm{Raz}^{4}$ \\ Igal Leibovitch' \\ 'Oculoplastic and Orbital Institute, \\ Department of Ophthalmology, \\ Sackler Faculty of Medicine, Tel \\ Aviv University, Tel Aviv, Israel; \\ ${ }^{2}$ Department of Plastic Surgery, Assaf \\ Harofeh Medical Center, Tzrifin, Israel; \\ ${ }^{3}$ Infectious Disease Unit, ${ }^{4}$ Pathology \\ Unit, Tel Aviv Sourasky Medical \\ Center, Sackler Faculty of Medicine, \\ Tel Aviv University, Tel Aviv, Israel
}

\begin{abstract}
A 44-year-old woman, who had undergone gold-weight implantation due to facial palsy and lagophthalmos, arrived at the ophthalmology ward with eyelid swelling and erythema, which rapidly deteriorated under intravenous antibiotics to a necrotic process involving the periocular tissues, the eye, and the anterior orbit. Despite prompt removal of the gold weight, the patient's ocular and systemic condition continued to deteriorate, necessitating evisceration and debridement of necrotic tissue. Cultures showed growth of Staphylococcus epidermidis, Staphylococcus capitis, Candida glabrata, and Candida albicans, and histopathology demonstrated an acute nonspecific necrotizing panophthalmitis. Later on, the patient was admitted to a plastic surgery ward with recurrent severe burns of her thigh, which were highly suggestive of being self-induced, raising the possibility of self-induced damage.
\end{abstract}

Keywords: evisceration, factitious disorder, lagophthalmos

\section{Introduction}

Gold weight loading of the upper eyelid is a procedure performed for the treatment of paralytic lagophthalmos. The incidence of complications is reported to range from $0.5 \%$ to $61 \%{ }^{1}$ and includes poor cosmesis, migration, extrusion, allergy, and astigmatism. ${ }^{2}$ We report herein what seems to be the first case of gold weight implantation complicated by a severe necrotic process, necessitating extensive excision of eyelid tissue as well as evisceration of the involved eye.

\section{Case report}

A 44-year-old Caucasian woman with a history of right-sided facial paralysis following removal of an acoustic neuroma presented to our clinic with lagophthalmos. Due to continued keratopathy, implantation of a gold weight was then performed in her right upper eyelid. The immediate postoperative period was unremarkable.

On postoperative day 11, the patient presented with right eyelid swelling and erythema, as well as limitation of abduction, suggesting a possibility of orbital cellulitis. The patient was treated with intravenous cefuroxime. Within a few hours, a complete limitation of gaze was noted, followed by an afferent pupillary defect and necrosis of the lower eyelid, of the upper eyelid margins, and of the anterior ocular segment. Computed tomography scan was unremarkable apart from swelling of superficial tissues. The patient was taken to the operating room where the gold weight was removed through the previous skin incision. On exploration, necrosis was noted in the upper eyelid tarsus, lower eyelid, limbus, and conjunctiva (Figure 1). The cornea was opaque and a dense cataract was noticed. A full thickness biopsy of the eyelid revealed necrotic
Correspondence: Roy Schwartz Tel Aviv Souraski Medical Center, 6Weizmann Street, Tel Aviv 64239, Israel Tel +972 36974000

Email royschwartz@gmail.com 


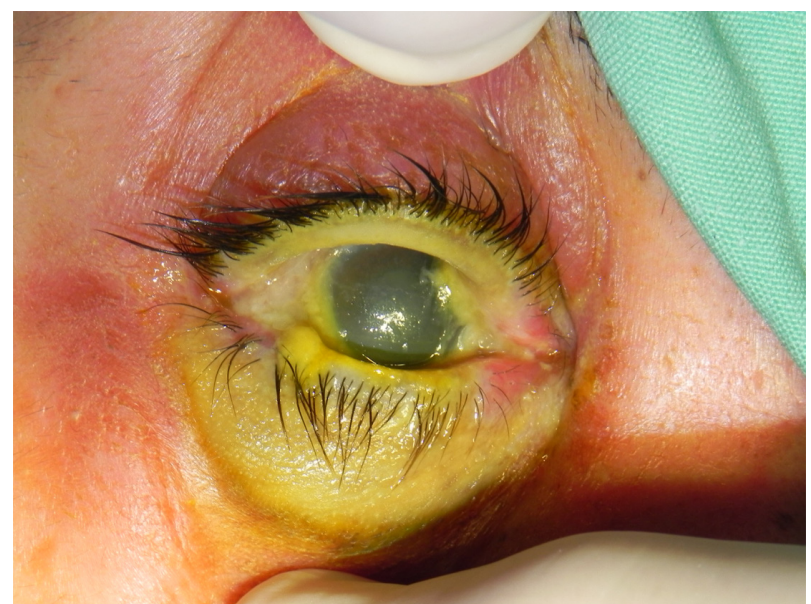

Figure I Ischemia of lower eyelid, upper eyelid margins, and anterior segment seen 24 hours after admission.

conjunctiva and skin, with diffuse acute inflammatory reaction. Gram stain revealed gram-positive cocci, and cultures grew Staphylococcus capitis.

The antibiotic regimen was changed to intravenous vancomycin as well as piperacillin/tazobactam, which resulted in stabilization. Visual acuity was light perception only. A transcranial Doppler was performed to rule out an ischemic etiology, and showed normal flow in both ophthalmic arteries.

Four days following removal of the gold weight, the patient developed fever of $38^{\circ} \mathrm{C}$, chills, tachycardia, and extreme restlessness, suggesting systemic involvement, as well as thinning and necrosis of the sclera, worsening of eyelid swelling and necrosis (Figure 2). An axial computed tomography scan with contrast material demonstrated air bubbles under the eyelid as well as intraconal fat haziness,

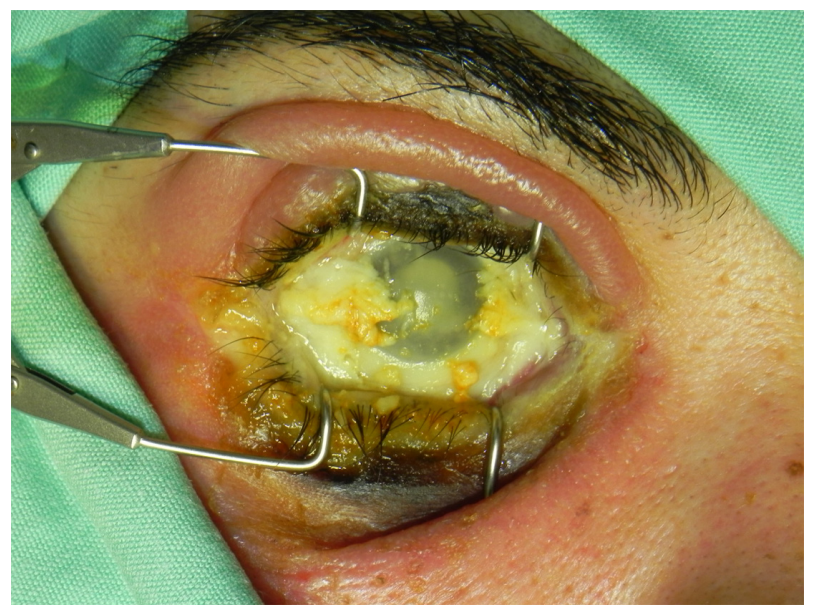

Figure 2 Further necrosis of eyelid, as well as scleral necrosis, seen 4 days after gold weight removal. implying an infectious or inflammatory process involving the anterior and posterior parts of the orbit. The patient was taken again to the operating room where evisceration and debridement of necrotic tissue were performed (Figure 3). Histopathologic examination revealed acute nonspecific necrotizing panophthalmitis (Figure 4). Direct smear showed gram-positive cocci, and cultures grew Staphylococcus epidermidis, Candida albicans, and Candida glabrata.

Clindamycin and voriconazole were added. The patient's systemic condition continued to deteriorate, including fever, urinary retention, desaturation, and acidosis, and she was admitted to the intensive care unit where her systemic condition stabilized. The antibiotic regimen was changed to vancomycin, meropenem, and fluconazole. With this treatment, the patient's condition gradually improved and her ocular condition remained stable. On repeat follow-ups, a severe contracted socket was seen (Figure 5).

A year later, this patient was admitted to the plastic surgery ward in another hospital with a 5-month-old nonhealing third-degree chemical burn of her right anterior thigh. Wound debridement under local anesthesia failed due to lack of patient cooperation. Therefore, a second operation with complete debridement was done under general anesthesia. A split thickness skin graft was used to cover the defect and the wound was dressed with a negative pressure dressing.

By postoperative day 2, the negative pressure dressing was found with tears over its surface and could not maintain vacuum. A dressing change was made, revealing a partially taken skin graft. The patient's thigh was dressed with a twice daily Sulfamylon wet-to-dry dressing.

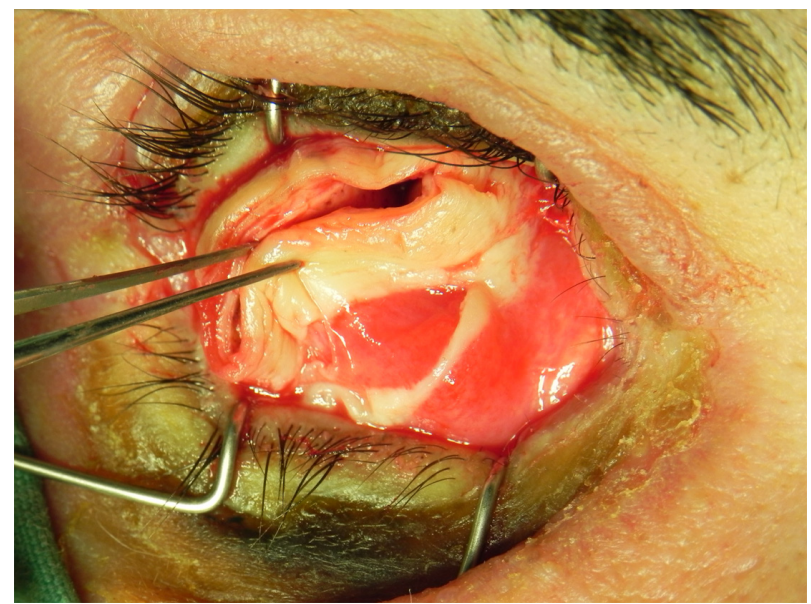

Figure 3 Photograph taken during evisceration surgery showing the white, necrotic tissue. 


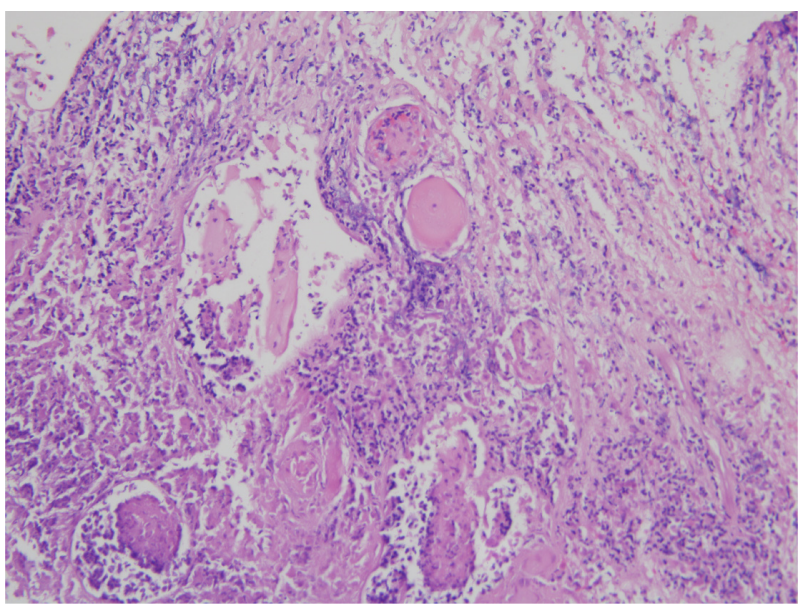

Figure 4 Histopathologic examination from evisceration (Hematoxylin and eosin stain, $\times 40$ ) revealing an acute nonspecific necrotizing inflammation involving the retina and uveal tissue (composed of necrotic segments), and a dense inflammatory infiltrate composed mainly of polymorphonuclear cells.

The postoperative period was remarkable, with the patient experiencing tenderness, itching, and unrest. At postoperative day 11, instead of full closure of the wound, a full thickness third degree burn was noticed once again with no signs of infection. The patient underwent a further operation the next day under general anesthesia for debridement and placement of partial thickness skin graft. A day after the surgery, the patient tore off both the dressing and the skin graft, necessitating a third skin grafting. This time, the patient was put into a full length cast for protection and immobilization.

Due to an impression of self-induced damage and a high suspicion of a factitious disorder during the latter hospitalization, a psychiatric consult was made, but concluded that a

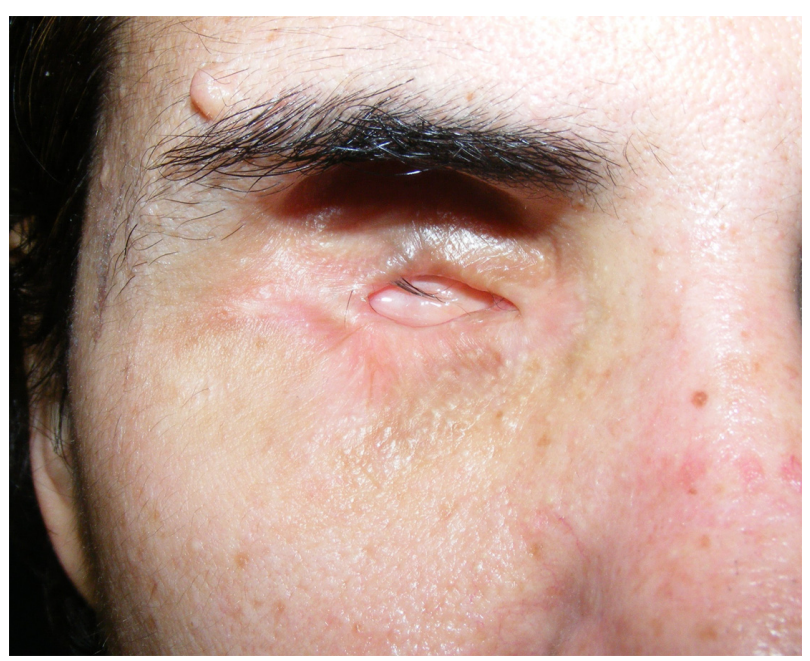

Figure 5 The contracted socket as seen on follow-up of the patient 6 months post-evisceration. diagnosis of a factitious disorder could not be made without direct evidence of self-mutilation.

\section{Discussion}

Of the many case reports and case series describing complications after implantation of an eyelid gold weight, none presented as dramatically as in the case of this patient.

The rapidity in which the patient deteriorated, the findings of gas on computed tomography, as well as the histopathologic findings of a dense inflammatory infiltrate composed predominantly of polymorphonuclear leukocytes and of thrombotic blood vessels suggested an acute infection as the etiology of this process. S. capitis and S. epidermidis, as other coagulase-negative staphylococci, are known to cause foreign-body-associated infections. However, a dramatic clinical course, as in our patient, would be extremely unusual. Candida species, which were isolated only from tissues obtained in the evisceration surgery and not in the preliminary cultures, are also uncommon pathogens in such processes and are likely to have been contaminants.

Although the clinical course was consistent with necrotizing fasciitis involving the eyelid with a severe systemic septic syndrome, the histopathologic findings did not demonstrate involvement of the fascia, and the typical culprits of necrotizing fasciitis (eg, Streptococcus pyogenes, Enterobacteriaceae, anaerobic bacteria, methicillin-resistant Staphylococcus aureus) were not isolated. Eyelid involvement of necrotizing fasciitis is rare, with only 58 cases reported between 1956 and 2006. The process usually spares vascularized structures, namely, the conjunctiva, tarsus, and eyelid margins, which were not spared in our case. ${ }^{3}$

Allergy to gold is a known complication of gold weight eyelid implantation. However, it usually manifests as contact dermatitis, and histology reveals chronic lymphocytic infiltrates, matching a type IV hypersensitivity reaction; whereas, in our case, the infiltrate included mostly polymorphonuclear leukocytes. ${ }^{4}$ Our patient kept deteriorating after removal of the gold weight, further negating contact with the weight as the inciting factor.

In factitious disorders, patients intentionally produce signs of medical disorders and misrepresent their histories and symptoms. The disorders appear more frequently in females. In a 1951 article in The Lancet, Richard Asher coined the term "Munchausen syndrome" to refer to a syndrome in which patients embellish their personal history, chronically fabricate symptoms to gain hospital admission, and move from hospital to hospital. ${ }^{5}$ Patients with the disorders simulate 
serious illness and seek and submit to unnecessary treatment, while to themselves and others they deny their true illness and, thus, avoid possible treatment for it. Ultimately, patients elude meaningful therapy by abruptly leaving the hospital or failing to keep follow-up appointments. ${ }^{6}$

The unique pattern of injury to the patient's eye, as well as the repeated burns and behavior, suggest that the patient might suffer from factitious disorder, a condition that is, unfortunately, difficult to diagnose.

\section{Disclosure}

The authors report no conflicts of interest in this work.

\section{References}

1. Tower RN, Dailey RA. Gold weight implantation: a better way? Ophthal Plast Reconstr Surg. 2004;20(3):202-206.

2. Bladen JC, Norris JH, Malhotra R. Indications and outcomes for revision of gold weight implants in upper eyelid loading. $\mathrm{Br} \mathrm{J} \mathrm{Ophthalmol.}$ 2012;96(4):485-489.

3. Elner VM, Demirci H, Nerad JA, Hassan AS. Periocular necrotizing fasciitis with visual loss pathogenesis and treatment. Ophthalmology. 2006;113(12):2338-2345.

4. Doyle E, Mavrikakis I, Lee EJ, Emerson R, Rainey AJ, Brittain GP. Type IV hypersensitivity reactions to upper lid gold weight implants - is patch testing necessary? Orbit. 2005;24(3):205-210.

5. Asher R. Munchausen's Syndrome. Lancet. 1951;257(6650):339-41.

6. Kaplan BJ, Kaplan VA. Kaplan and Sadock's Synopsis of Psychiatry: Behavioral Sciences/Clinical Psychiatry. 9th ed. Philadelphia, PA: Lippincott Williams \& Wilkins; 2003:668-675.
Clinical Ophthalmology

\section{Publish your work in this journal}

Clinical Ophthalmology is an international, peer-reviewed journal covering all subspecialties within ophthalmology. Key topics include: Optometry; Visual science; Pharmacology and drug therapy in eye diseases; Basic Sciences; Primary and Secondary eye care; Patient Safety and Quality of Care Improvements. This journal is indexed on

Submit your manuscript here: http://www.dovepress.com/clinical-ophthalmology-journal

\section{Dovepress}

PubMed Central and CAS, and is the official journal of The Society of Clinical Ophthalmology (SCO). The manuscript management system is completely online and includes a very quick and fair peer-review system, which is all easy to use. Visit http://www.dovepress.com/ testimonials.php to read real quotes from published authors. 